\title{
Histological Study On The Testes of African Striped Ground Squirrel (Xerus Erythropus)
}

\author{
Korzerzer, R. M. ${ }^{1}$, Hambolu, J. O. ${ }^{2}$, Oladele, S. B ${ }^{3}$ and Suleiman, M. H'. \\ ${ }^{1}$ Department of Veterinary Anatomy, Federal University of Agriculture, Makurdi \\ ${ }^{2}$ Department of Veterinary Anatomy, Ahmadu Bello University of Zaria \\ ${ }^{3}$ Department of Veterinary Pathology, Ahmadu Bello University, Zaria
}

Accepted January, 2019 and Published February, 2019

\begin{abstract}
In this study, the testes of African striped ground squirrels (Xerus erythropus)obtained between the months of January and May were studied at histological level using Haematoxilin and Eosin stain, Masson Trichome stain and Periodic acid Schiff to examine the histo-architechure, collagen and glycogen localizations in the testes respectively. The results showed that the testes had finely arranged cells within the seminiferous tubules which were positive for both Masson-trichome and Periodic acid shiff by reacting to the blue and magenta colours respectively. However, the histoarchitechture of the testes did not show spermatogenic activity. In conclusion, the testes of African striped ground squirrel obtained between the months of January and May did not show detailed spermatogenic activity. This suggests that the squirrel is a seasonal breeding rodent with a dormant reproductive phase occurring around January to May. The testes also contains cells that express glycogen which suggests that energy in form of glycogen may be needed for the initiation of spermatogenesis.
\end{abstract}

Keywords: Histology, Glycogen, Collagen, Testes, Squirrel

*Corresponding author:

Email: rachemgy@yahoo.com

Tel: +2347037156525 


\section{INTRODUCTION}

The mammalian testes are paired and consists of highly coiled seminiferous tubules that produce spermatozoa, and of specialized cells called interstitial cells of Leydig that secret testosterone [5]. Each testis is covered with a thick fibrous capsule called tunica albuginea. The thick in-folding of the tunica albuginea at the posterior margin of the testis forms the mediastinum of the testis [1]. Histologically, the adult mammalian testis can be divided into two compartments, a seminiferous tubular compartment and an interstitial compartment. The tubular compartment consists of an outer layer (s) of peritubular myoid cells and an inner layer of seminiferous epithelium separated by an intermediate layer of acellular matrix or basement membrane [2]. The interstititial compartment consists of Leydig cells, immune cells such as macrophages and lymphocytes, and fibroblasts. In addition, it also contains blood and lymph vessels, nerves, and loose connective tissue. The tubular and interstitial compartments of the testis perform the functions of spermatogenesis and steroidogenesis, respectively [3]. The changes in testicular tissue of most animals is determined by two primary factors which include the age [1] and the season [3 and 2]. The aim of the present study was therefore to observe the histology of the testes of the nonbreeding adult male African striped ground squirrel (Xerus erythropus).

\section{Materials and Methods}

Ten apparently healthy adult male African striped ground squirrels (ASGS) (Xerus erythropus) were caught alive by use of traps around Zaria and environs between the months of January and May, 2017. The animals were conveyed in constructed iron cages to the Department of Veterinary Anatomy, Faculty of Veterinary Medicine, Ahmadu Bello University, Zaria, where the research was conducted. The parameters used for determining if the rodents had attained sexual maturity were anogenital distance [6], body weight and X-ray photographs of the hindlimbs to observe the epipyseal growth plates of the ASGS (Xerus erythropus). The research animals were acclimatized for a period of twenty-one days, during which period they were fed with groundnut, water melon, sweet potatoes, tomatoes and water ad libitum. Blood samples were analysed at the Parasitology Department, Faculty of Veterinary Medicine, Ahmadu Bello University, Zaria for haemoparasites. The body length (distance from the nose to the tip of the tail) of each animal was measured using a measuring thread and rule. The $\mathrm{X}$-ray photographs were taken at the imaging unit of Faculty of Veterinary Medicine, Ahmadu Bello University, Zaria using the conventional mobile $\mathrm{X}$-ray machine. The procedure involved placing the research animals on the $X$-ray table on ventral recumbecy with their limbs stretched out, and the X-ray beams passed on the limbs to obtain the caudo-cranial view of the limbs. The X-ray photographs were processed manually, almost immediately in order to produce a visible radiographic image that can be interpreted. The films were placed in a developer solution for sixty minutes, and then washed in a bath of free running water for fifteen minutes to remove excess chemicals, and then fixed in an acidic fixing solution to remove the undeveloped silver bromide crystals from the film emulsion and to re-harden the emulsion that was softened during the development process. The films were washed again in-order to remove residual chemicals such as the acid, thiosulphide and silver bromide and then dried on a drying hanger. The dried films were then placed behind the illuminator for viewing, and photographs were taken for interpretation using a digital camera (Samsung, SH100, 14.2 megapixels).

The body weights of the animals were obtained using a weighing balance (Fuji Keiryo 
weighing scale $\mathrm{K}-1,210 \times 210,200 \mathrm{~g}-8 \mathrm{~kg}$ ) and then euthanized with $30 \mathrm{mg} / \mathrm{kg}$ body weight of thiopental sodium anaesthesia given intramuscularly, and then placed on dorsal recumbency and a thoracolumber incision made up to the point of the pubic region, to expose the reproductive organs. The skin and superficial tissues were first reflected to obtain the testes, epididymis, ductus deferens, penis and the accessory sex glands. Then photographs of the organs were taken using a digital camera (Samsung SH100, 14.2 megapixels) and also weighed using an electronic balance (GG 1000 USA, with sensitivity 0.01).

\section{Ethical Approval}

Ethical approval was sought from the Care on Animal Use Committee (CAUC), Ahmadu Bello University, Zaria with approval number ABUCAUC/2018/025.

\section{Histological studies}

After weighing the organs, each was labelled and fixed for 48 hours in $10 \%$ neutral buffered formalin [7]. Dehydration was done through a series of graded alcohol $(70 \%, 80 \%, 90 \%, 95 \%$ and $100 \%)$. Then cleared with xylene and infiltrated with molten paraffin wax. Sections of $4 \mu$ thick were made from the embedded tissues using a microtome (Leica, Model RM 2125RTS, Biosystems, Made in China).

\section{Staining of sections}

\section{Haematoxilin and Eosin (H and E)}

The sections of the tissues were mounted on grease free glass slides and the Haematoxylin and Eosin (H\&E) staining for routine histological studies was carried out according to the protocol of Romeis Mikroskopishe Technik [4]. The sections were immersed in Haematoxilin solution for 7 minutes, which was done immediately after removal from distilled water. This was washed in running tap water for 20 minutes and then passed into $50 \%$ ethanol for 2 minutes. The sections were then counterstained with eosin for 3 minutes and immersed in 2 changes of $96 \%$ ethanol, isopropanol and xylol in that order for 2 minutes each. Tissues were mounted using oil mountant and cover slips. The slides were then examined using a light binocular microscope, Made in China at different magnifications $(\times 40, \times 100$, $\times 250$ and $\times 400$ ). Photomicrographs of the tissues were obtained by using an Amscope MT digital camera for microscope MD 500, connected to a Compact Presario computer.

\section{Masson and Trichome}

Masson trichome stain was employed to study the distribution of collagen fibres. The sections were taken to water and covered with Weigert's Haematoxilin for 20 minutes at RT. Then covered with cytoplasmic stain for 2 minutes and covered with dodeca-molybdophosphoric acid hydrate for 5 minutes. The sections were then covered with the light green stain for 2 minutes, and washed again in water. Sections were dehydrated, cleared and mounted in Distyrene plasticizer xylene (DPX), which is a permanent mountant that protects the stained sections from fading and dust, and also functions to increase the refractive index of the slides to make the sections appear clearer. The slides were then examined using a light microscope at varying magnifications $(\times 40$, $\times 100, \times 250$ and $\times 400$ ). Photomicrographs of the tissues were obtained by using an Amscope MT digital camera for microscope MD 500, connected to a Compact Presario computer.

\section{Periodic Acid Schiff}

Periodic acid schiff (PAS) was the special stain used for studying glycogen distribution within the reproductive organs. The cut sections of $4 \mu \mathrm{m}$ were placed in water and then covered with periodic acid for 5 minutes. The sections were then covered with Schiff reagent for 15 minutes at RT ${ }^{\circ} \mathrm{C}$ and covered with Haematoxilin for 2 minutes. Sections were dehydrated, cleared and mounted in DPX mountant. The slides were later examined using 
a light microscope at varying magnifications $(\times 40, \times 100, \times 250$ and $\times 400)$. Photomicrographs of the tissues were obtained by using an Amscope MT digital camera for microscope MD 500, connected to a Compact Presario computer.

\section{RESULTS}

The testes of adult male African striped ground squirrel migrates from the scrotum and move closer to the abdomen during the inactive reproductive phase (Plate1). Grossly, the testes appear dark red in the non active phase instead of the white appearance during the active reproductive phase observed in most mammals. Histologically, the testes comprised of seminiferous tubules which contained numerous spermatogonia/sertoli cells (Plate 3). The cells appeared as finely arranged cells within the seminiferous tubules of the testis, and stained blue with Masson Trichome stain (Plate 4) and magenta with Periodic acid Schiff (Plate 5). The histology of the testes did not show clear distinct spermatogenic activity. The major significant finding in this study is the absence of spermatogenic activity and apparently inactive testicular tissue of the testes of adult African striped ground squirrel between January and May.

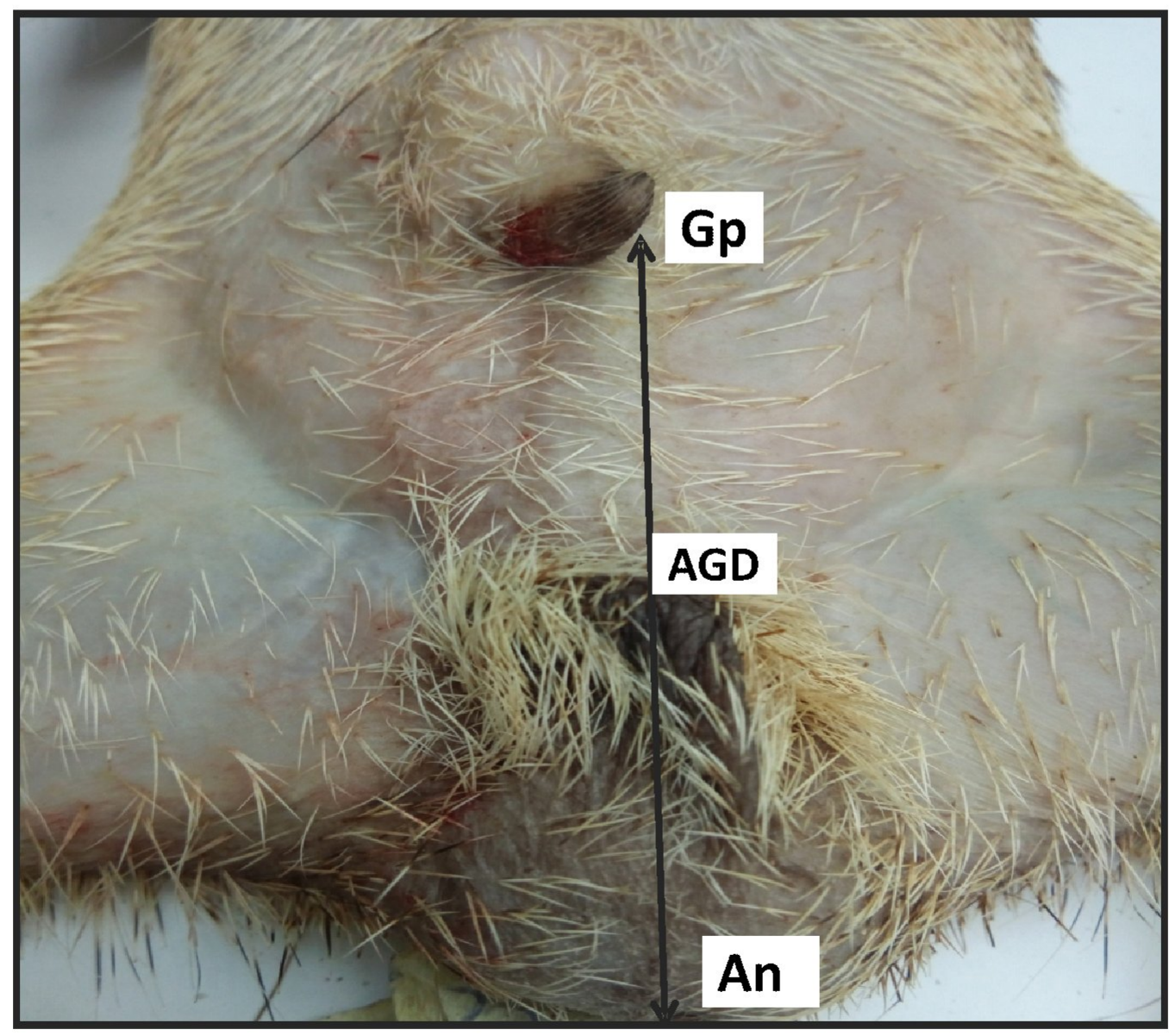

Plate 1: Photograph of the genital area of the ASGS (Xerus erythropus) showing the anogenital distance (AGD) indicated by the black arrow which extends from the genital projection (Gp) to the anus (An). 


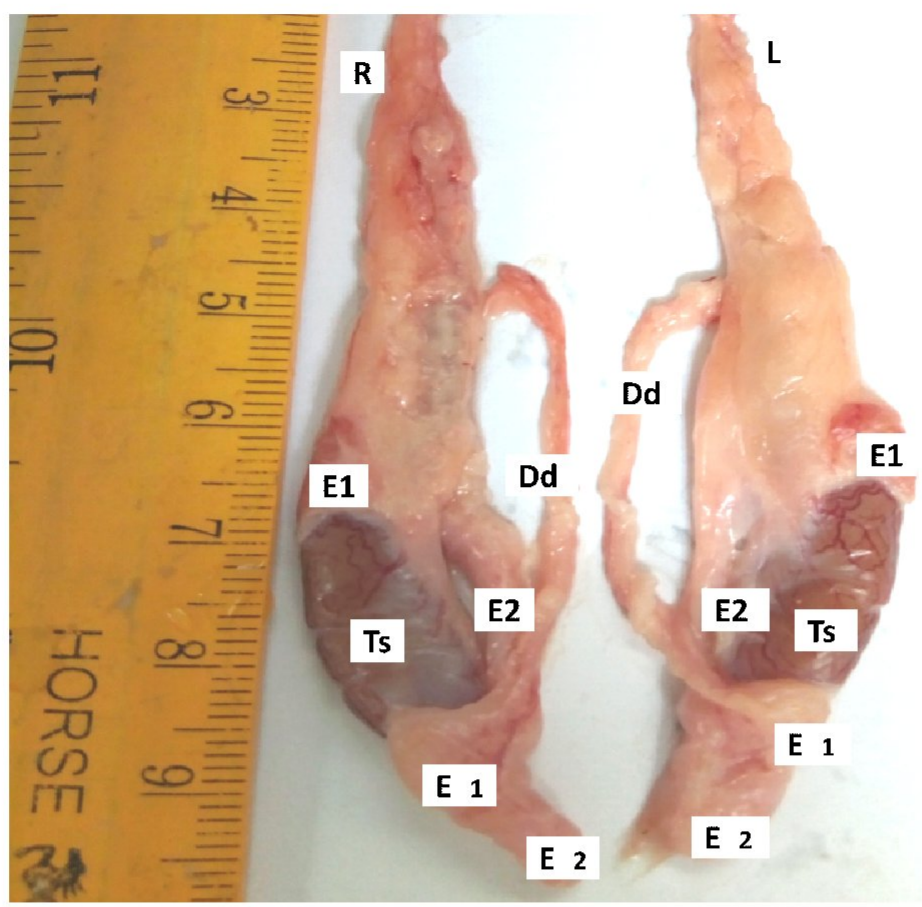

Plate 2: Photograph of both right (R) and left (L) testes of the ASGS (Xerus erythropus) showing the Testis (Ts), catput epididymis (E1), corpus epididymis (E2), fixed caudal epididymis (E3), free caudal epididymis $\left(\mathbf{E 3}_{2}\right)$ and ductus deferens (Dd).

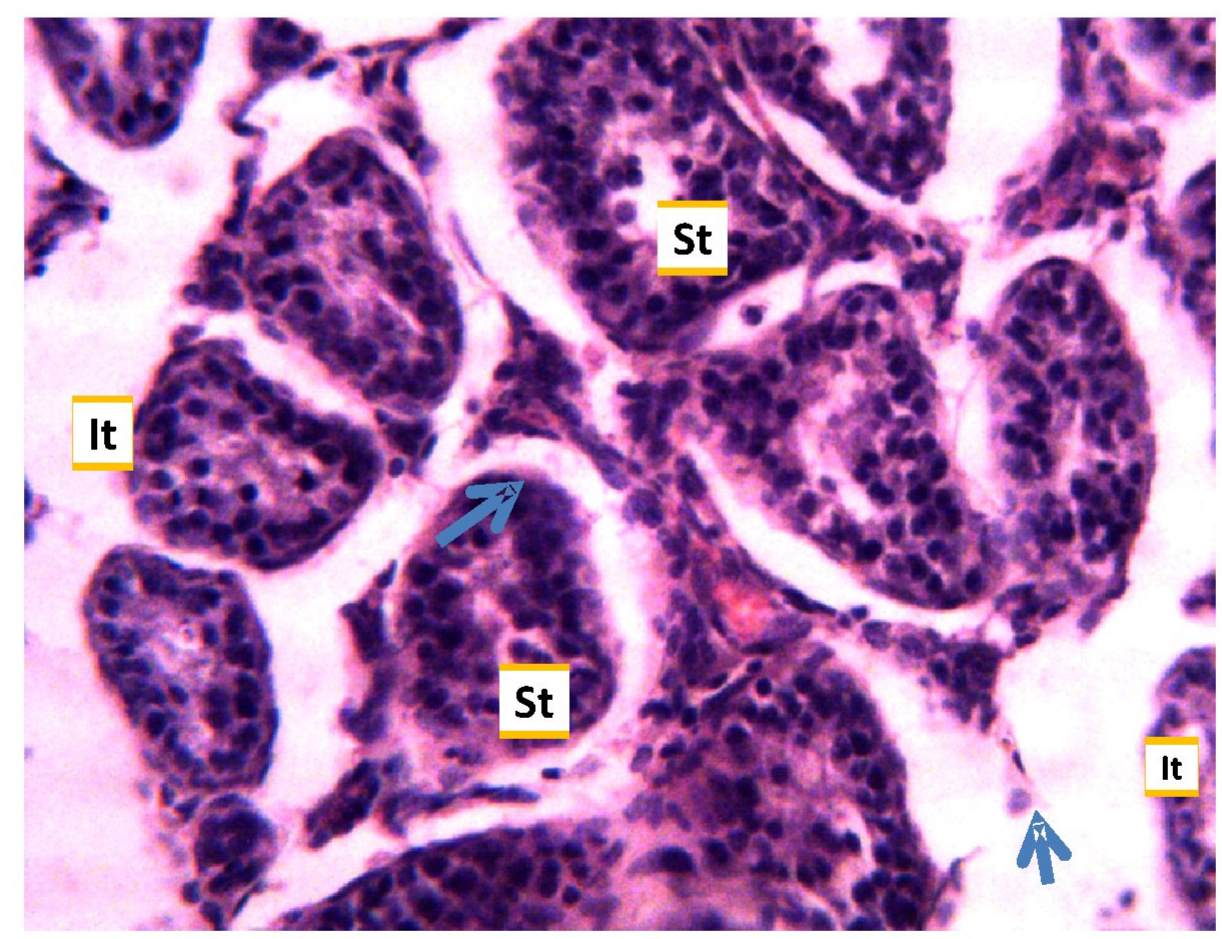

Plate 3: Photomicrograph of the left testicular tissue of ASGS (Xerus erythropus) showing the seminiferous tubules (St), the leydig cell tissue shown by blue arrows. Note the loose connective tissue in the spaces between individual seminiferous tubules (It). $\mathrm{H} \& \mathrm{E} \times 250$. 


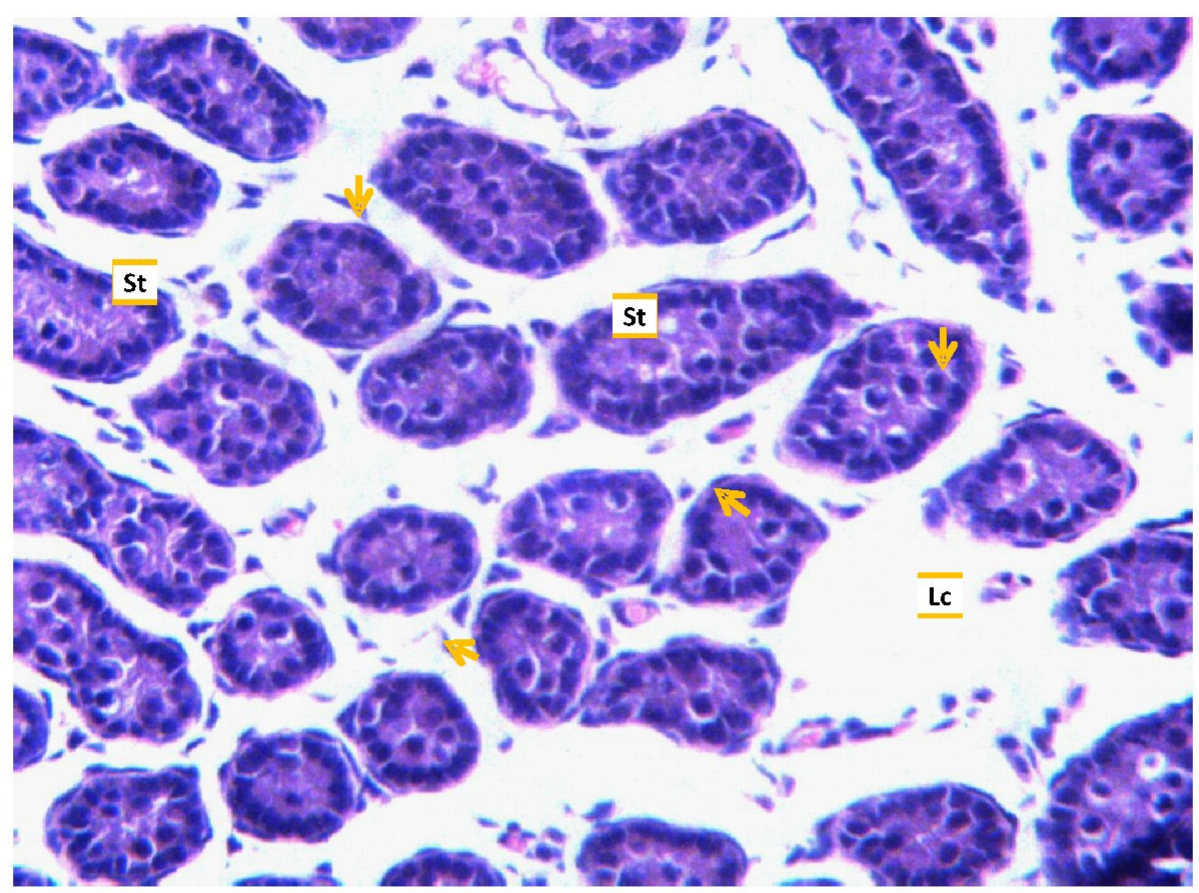

Plate 4: Photomicrograph of the left testicular tissue of ASGS (Xerus erythropus) showing the seminiferous tubules (St), the leydig cell (Lc). Note the positive stained cells shown by yellow arrows. Masson-Trichome $\times 100$.

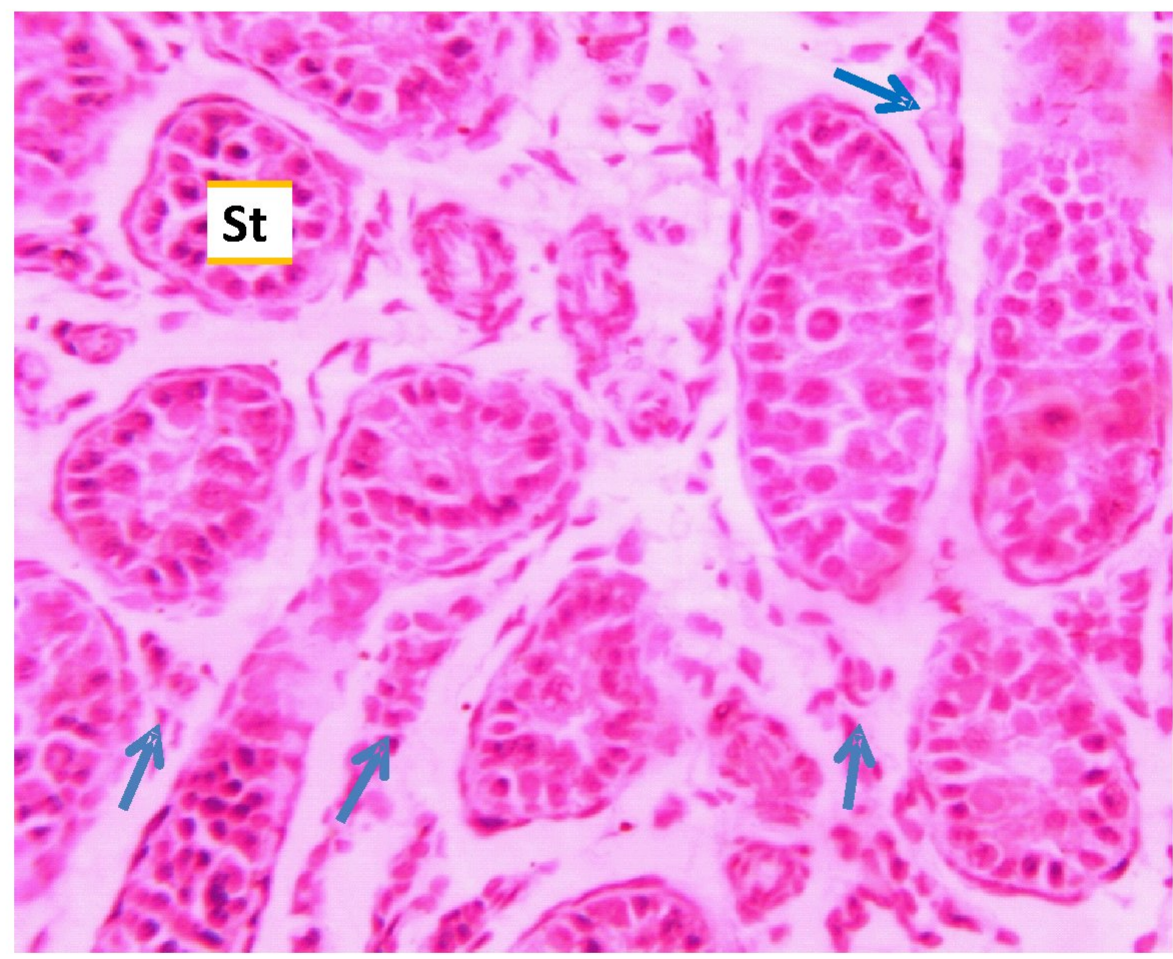

Plate 5: Photomicrograph of right testicular tissue of ASGS (Xerus erythropus). Note the positive reactive cells (sertoli cells/spermatogonia) within the seminiferous tubules (St), the leydig cells are shown by blue arrows. PAS $\times 250$. 


\section{Discussion}

The histological appearance of the testes was not consistent with that observed in other mammals which may be due to the inactive reproductive phase observed in seasonal breeding rodents. In the seminiferous tubules, the sertoli cells and spermatogonia demonstrates the presence of glycogen. This finding suggests that energy is required for most cellular processes to occur, and these cells possess the energy required for the initiation of spermatogenesis.

The histo-architecture of the testes of African striped ground squirrels studied did not show active sign of spermatogenesis, and this infers that the African striped ground squirrel may be a seasonal breeder, experiencing periods of sexual dormancy during periods of unfavourable environmental conditions, although more studies should be carried out in the rainy months to ascertain this state in the rodent.

\section{CONCLUSION}

The present study in the African striped ground squirrel (Xerus erythropus) suggets that the rodent is a seasonal breeder presenting an inactive reproductive phase between January and May. The testes in the inactive phase differs both grossly and histologically with that of other mammals. The testicular sac was flabby and testes had migrated towards the abdomen. The positive reaction for glycogen suggests that glycogen may be necessary within the testes to provide the needed molecules of ATP during cellular processes within the testes, including the initiation and maintainance of spermatogenesis.

\section{References}

[1]Gerzilov, V., Bochukov, A., Penchev, G. and Petrov, P. (2015). Testicular development in the Muscovy duck (Cairina moschata). British Journal of Veterinary Medicine, (online), DOI: $10.15547 / \mathrm{bjvm} .897$.

[2]Islam, F., Ishishita, S., Uno, Y., Mollah, B., Skrikulnath, K. and Matsuda, Y. (2013). Male hybrid sterility in the mule duck is associated with meiotic arrest in primary spermatocytes. Journal of Poultry Science, 50, 311-320.

[3]Leska, A., Kiezun, J., Kaminska B. and Dusza, L. (2012). Seasonal changes in the expression of the androgen receptor in the testes of the domestic goose (Anser anser $f$. domestica). General Comparative Endocrinology, 179(1), 63-70.

[4]Mulisch, M., Welsch, U., Aescht, E. and Romeis, B. (2010). Romeis Mikroskopische Technik. $\left(19^{\text {th }}\right.$ ed.) Heidelberg Spektrum publishers. Pp 330-340.

[5]Nieschlag, E. and Behre, H. (2000). Andrology, Male reproductive Health and Dysfunction, $2^{\text {nd }}$ edition, Springer, Berlin.

[6]Sacramento, T. I., Sinabaragui, O. S., Aizoun, F. and Farougou, S. M. (2013). Determination of grasscutter age (Thyronomys swinderianus) from anogenital distance. Journal of Applied

Biosciences, 62:4637-4643

[7] Onuoha, E. O. (2010). A Practical Guide for the Preparation of $\mathrm{Histological}$, Histopathological and Cytological Slides. Fepam ventures, Nigeria. 